\title{
Design and Implementation of a Multi-Threaded TMN Agent System
}

\author{
Sang-Kwon Lee \\ KAIST, KOREA \\ sklee@ camars.kaist.ac.kr \\ Seong-Bae Eun \\ Hannam University, KOREA
}

\author{
Minhee Cho \\ KAIST, KOREA \\ mhcho@camars.kaist.ac.kr \\ Hyunsoo Yoon \\ KAIST, KOREA
}

\author{
Hyeon-Ju Yoon \\ Ewha Womans University, KOREA \\ juyoon@mm.ewha.ac.kr \\ JungWan Cho \\ KAIST, KOREA
}

\begin{abstract}
A telecommunications management network adopts the OSI manager-agent model in which an agent system plays more important role than a manager system. Because the existing agent systems sequentially perform a CMIS operation on selected managed objects, it cannot guarantee quick response time when many managed objects are selected. In this paper, we propose a multi-threaded process structure for the agent system which performs CMIS operations concurrently on managed objects. We implemented our agent system using OSIMIS-4.0 TMN platform in Solaris. We measured response time to a CMIS operation to evaluate performance of our system. Experimental results show that performance of our system is not so good when network resources reside within the agent system, but very good when network resources reside outside the agent system.
\end{abstract}

\section{Introduction}

A TMN(Telecommunications Management Network) [7] makes use of the OSI concepts and protocols and adopts the OSI manager-agent model (Figure 1) [4]. It comprises a manager system which plays a manager role, an agent system which plays an agent role, and network resources which are the main concern of management [1, 6, 4]. Manager process invokes CMIS(Common Management Information Service) [2] operations on managed objects to monitor and control network resources. A managed object represents a management view of a network resource and communicates with the resource using various communication mechanisms such as RPC(Remote Procedure Call), IPC(Interprocess Communication) or system call.

The OSI network management has a characteristic that the complexity lies in the agent system as opposed to the manager system $[1,6]$. When an agent system receives an operation request from a manager system, it performs the operation on the managed objects which are selected by scoping. It takes long time to perform the operation on the managed objects due to accumulated communication delays. While a managed object waits for the response from the resource, it could result in blocking, a situation in which the agent system cannot process another operation request.

Many researches have been done on the development of an agent system $[5,8,9,10,12]$. The agent system of OSIMIS $[8,10]$ is a single-threaded UNIX process which uses an asynchronous IO technique to avoid blocking but not so effective. When it performs a CMIS operation it sequentially performs the operation on the selected managed objects, and this poses a serious problem of having very long response time to a CMIS request. Shim [12] proposed a multi-threaded UNIX process for the agent system to process more than 2 operations at the same time. When it performs a CMIS operation, however, it sequentially performs on the selected managed objects, so it has the same problem of having long response time to a CMIS request as OSIMIS. Furthermore, it did not resolve mutual exclusion problem between threads.

In this paper, we propose a new multi-threaded process structure for an agent system which does not get blocked and efficiently performs CMIS operations. We implemented the proposed system with the OSIMIS-4.0 TMN platform using $\mathrm{C}++$ programming language in Solaris operating system. Experimental results show that performance of our system is not so good when network resources reside within the agent system, but very good when network resources reside outside the agent system.

This paper is organized as follows. In section 2, we re-

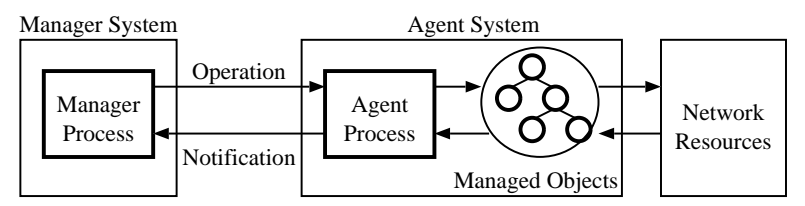

Figure 1. Manager-Agent model 
view components of an agent system and existing agent systems. In section 3, a new process structure is proposed for an agent system, which employs multiple threads. In section 4, the proposed system is evaluated in terms of response time to a CMIS operation. Finally, conclulsions are given in section 5 .

\section{An Agent System}

\subsection{Components and characteristics of an agent system}

In general, the management process for an agent system consists of three modules: coordinator, agent, and managed objects $[8,10,12]$. The coordinator passes control to a proper module whenever events (a CMIS operation request from the manager system, an event report from network resource, and a timer interrupt within the agent system) occur. The agent receives a CMIS request via the coordinator, selects target managed objects through scoping and filtering, relays the request to the selected managed objects, and forwards results from managed objects to the manager system. The managed object receives a CMIS request via the agent, translates the request into a command which the network resource can understand, and sends the command to the network resource. When the managed object receives a reply from the network resource, it translates the reply into a standard format and sends the reply to the manager system. The managed object receives an event report from the network resource, and its polling routine is called at regular intervals by the coordinator.

A managed object communicates with a network resource with various mechanisms according to the position of the resource: a system call is used if the resource resides within the operating system kernel, IPC if in another user space, and RPC if in the remote system. After the managed object invokes one of them, it waits for the response. M-GET, M-SET, M-ACTION, and M-DELETE operations may be performed on many managed objects through scoping and filtering [2, 3]. If RPC, for example, is sequentially called for each managed object, it will take long time to perform the operation due to an accumulated waiting time of RPC calls. Also, while a managed object is waiting for a response from a network resource, it could result in a blocking, a situation in which the entire agent system waits for the reponse. If a blocking occurs, the agent system cannot process incoming CMIS requests or incoming event reports.

\subsection{Previous Works}

OSIMIS is a platform for the development of management applications based on OSI $[8,10]$. OSIMIS provides an OSI agent engine called GMS(Generic Agent System), which is a single-threaded UNIX process and uses an asynchronous IO technique to avoid blocking [13, 14]. But the asynchronous IO provided by OSIMIS has a limitation that it is available only through file descriptor, so it cannot be used for the general request-response paradigm such as RPC/IPC/system call (all of these are synchronous IO). Since GMS is a singled-threaded process, the entire agent system gets blocked when a single managed object does. The asychronos IO provided by OSIMIS, therefore, cannot effectively avoids blocking. When many managed objects are selected by a CMIS operation, the GMS sequentially performs the operation on the managed objects. If the managed objects synchronously communicate with the network resource, the accumulated waiting time will be very long.

Shim proposed a multi-threaded UNIX process for an agent system [12]. Because Shim's system forks a new thread to service a CMIS request, the entire system does not get blocked. When many managed objects are selected by a CMIS operation, the operation is sequentially performed on the managed objects just like OISMIS. So, the same problem of having long response time exists. Since Shim's system can simultaneously service more than 2 operations, it can happen that more than 2 threads can simulataneously access the same managed object as shown in Figure 2. If there is no synchronization between threads, the integrity of the management information held in the managed object could be destroyed. But, Shim does not give any comment on the mutual exclusion problem.

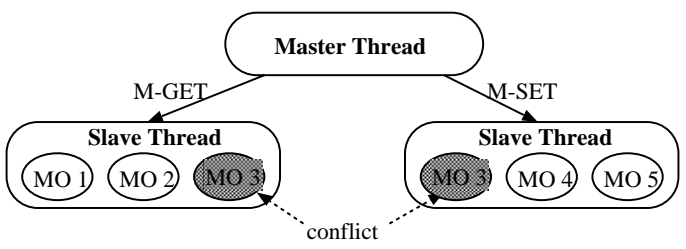

Figure 2. When the agent system performs two operations simultaneously

\section{Design and Implementation of an Agent Sys- tem}

\subsection{Process structure of an agent system}

Figure 3 shows the proposed process structure of an agent system which consists of multiple threads. Threads belonging to the process share information through global memory.

One coordinator thread exists within the agent system and runs until shutdown of the system. Whenever an event such as a CMIS request, an event report, or a timer interrupt occurs, the coordinator thread forks a thread (agent thread or MO thread) and passes control to the appropriate module. 


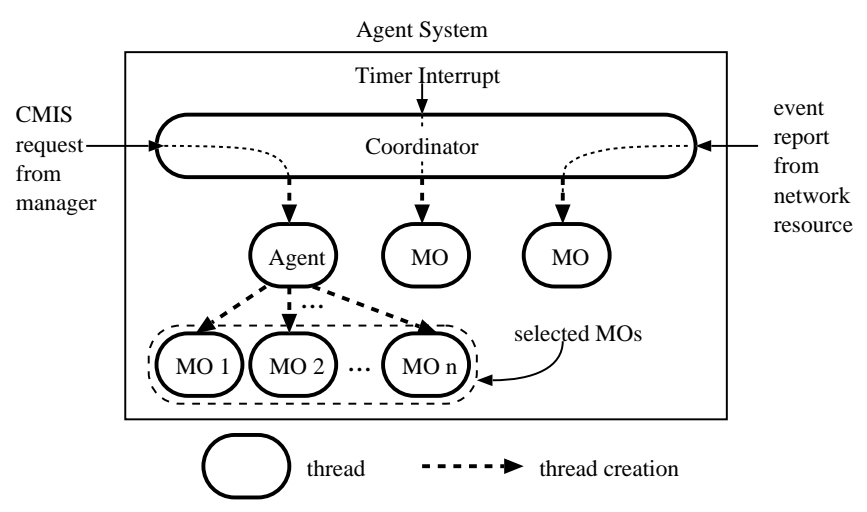

Figure 3. Process structure of an agent system

An agent thread is created by the coordinator thread to service a CMIS request. When it receives one of the MGET, M-SET, or M-ACTION operation request, it forks an MO thread for each managed object, performs the operation concurrently, and waits until all MO threads have finished. Although an M-DELETE operation can select many managed objects through scoping, we don't create thread for this operation. The reason is that it is not requested as frequently as M-GET/M-SET/M-ACTION, and that it is not required the operation be performed quickly.

An MO thread which is created by the agent thread communicates with the network resouce using synchronous IO (system call, IPC, or RPC), sends the result to the manager, and terminates its execution. An MO thread which is created by the coordinator thread handles an event report or a timer interrupt.

The proposed agent system has the following characteristics: First, because a CMIS operation is serviced by a number of threads, it allows the agent system to send response more quickly. Second, while some of MO threads get blocked during communication, the coordinator thread can handle new event by simply creating a new thread. So, there is no blocking. Finally, synchronous IO techniques (system call/IPC/RPC) makes it easy for a programmer to write applications.

\subsection{Thread manager}

The proposed system can simultaneously service more than 2 CMIS requests. In Figure 4, a managed object (MO 3 ) is selected by 2 CMIS requests (M-GET and M-SET), and $2 \mathrm{MO}$ threads have an access to it. This situation may result in information loss which is kept for the managed object.

In this paper, we use a thread manager to resolve a mutual exclusion problem between MO threads. The thread

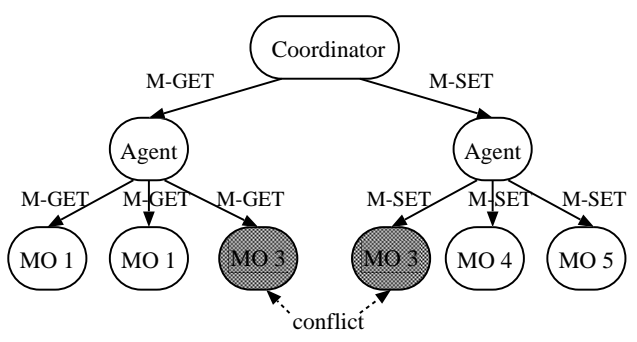

Figure 4. When 2 operations are simulataneously serviced in the agent system

manager keeps information on managed objects and guarantees that only one MO thread has an exclusive access to a given managed object. Before an MO thread performs a CMIS operation on a managed object, it requests the thread manager to acquire a lock on the managed object. After the thread has finished performing the operation, it requests the thread manager to release the lock. If the lock request is not allowed by the thread manager, the MO thread must wait until the request is allowed. In the later time, if another thread releases the lock on the same managed object, the thread manager selects an arbitrary one among pending threads, and allows the selected thread to perform an operation on the managed object.

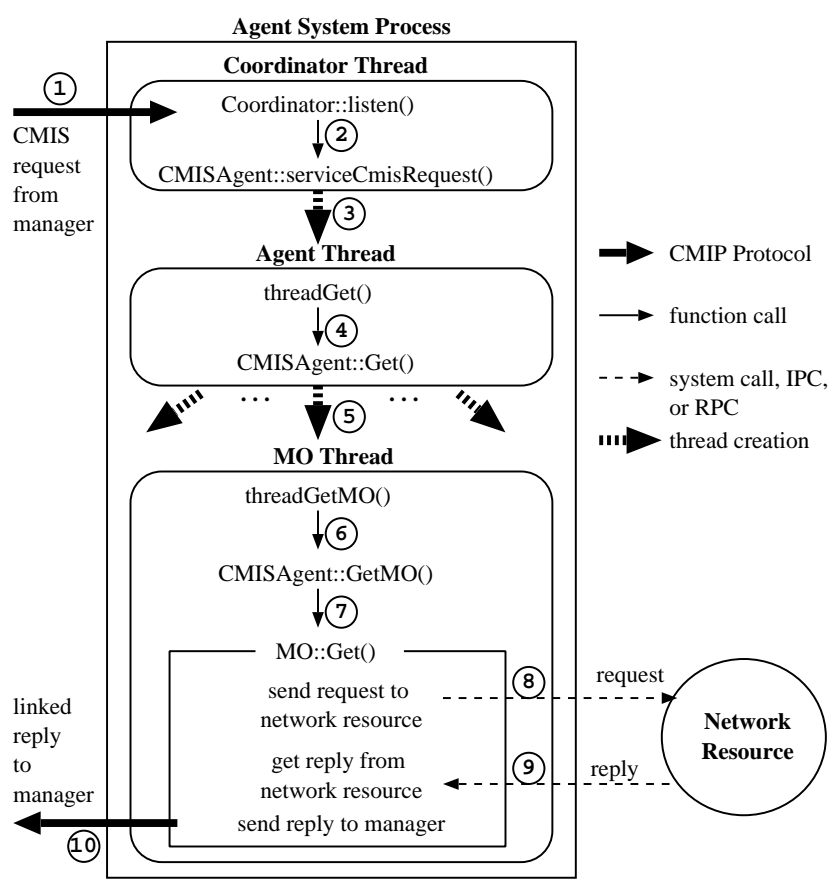

Figure 5. An overall flow of the agnet system (in the case of M-GET operation) 


\subsection{Implementation}

We implemented the proposed system using $\mathrm{C}++$ programming language in Solaris operating system with OSIMIS-4.0 TMN platform which provides CMIP protocol, agent API, manager applications, and GDMO compiler. We used ISODE(ISO Development Environment) 8.0 [11] for OSI protocol stack and pthread library for multi-threading and mutual exclusion. The agent system was implemented by modifying GMS source codes including: Coordinator, CMISAgent, and $M O$ class files, which respectively implement the coordinator, the agent, and the managed object . Figure 5 shows an overall flow of the agent system in case an M-GET operation is arrived. The number on arrows indicates the execution ordering.

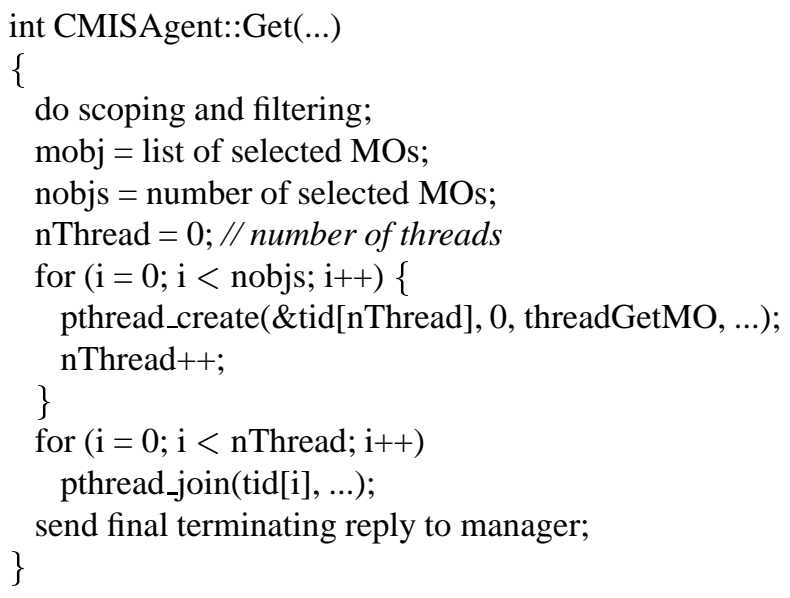

Figure 6. Create an MO thread for each managed object

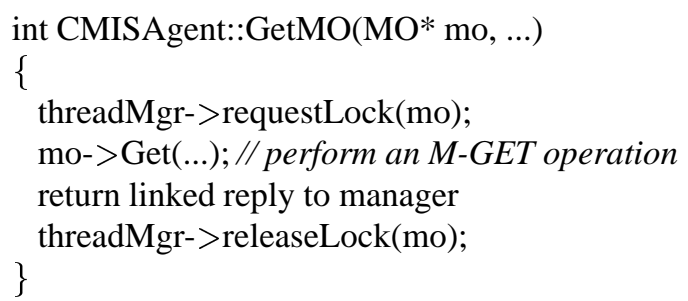

Figure 7. Perform an M-GET operation on the given managed object

When the coordinator receives an event report or a timer interrupt, it creates a thread in Coordinator::serviceWakeUp() and in Coordinator::readCommEndpoint_aux() respectively, and passes control to appropriate managed object. When the Coordinator class receives a CMIS request, it calls CMISAgent::serviceCmisRequest() which creates an appropriate agent thread according to the CMIS operation. Figure 6 shows a $\mathrm{C}++$-style pseudo code in which the agent thread creates an MO thread for each selected managed object to concurrently perform an M-GET operation. When the request is M-SET/M-ACTION, MO threads are created and terminated in a similar way. Figure 7 shows a pseudo code in which an MO thread performs an M-GET operation on a given managed object (mo). When the request is MSET/M-ACTION, the operation is performed in a similar way.

To resolve mutual exclusion problem, the thread manager uses pthread_mutex_t, a mutual exclusion variable type presented in pthread library, for each managed object. ThreadMgr::requestLock() is implemented by pthread_mutex_lock() and ThreadMgr::releaseLock() is implemented by pthread_mutex_unlock().

\section{Performance Evaluation}

In this section, we evaluate performance of the proposed system and compare it to that of OSIMIS. Performance evaluation has been done using various communication protocols: RPC, IPC, and system call.

\subsection{Communication protocol: RPC}

To evaluate performance of the agent system when RPC is used as a communication protocol, we constructed a management system which monitors the state of UNIX hosts connected to a network. Figure 8 shows the configuration of the management system used in experiments. OSIMIS TMN platform was installed both for the agent system and for the manager system. Using GDMO, we defined two managed object classes: unixHost which represents a UNIX host and subnet which represents a network. Whenever the agent receives an M-GET/M-SET/M-ACTION request, unixHost calls RPC to perform the operation, if needed.

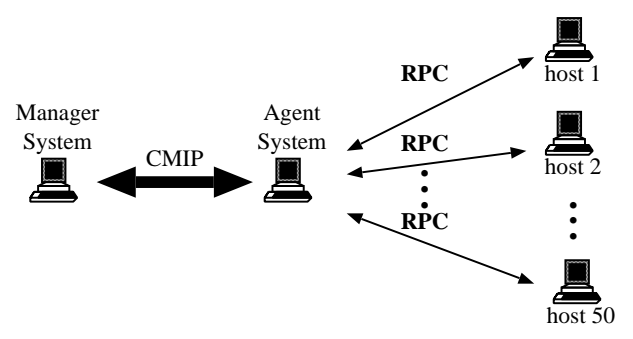

\section{Figure 8. System configuration when RPC is used as a communication protocol}

To measure the response time to a CMIS operation, we used manager applications provided by OSIMIS: mibdump, mset, and maction for an M-GET, M-SET, and M-ACTION operation, respectively [14]. We measured a response time as a difference between the time the agent system receives 
a CMIS operation and the time the agent system sends a final reply to the manager system. The proposed system is named for "Proposed", and that of OSIMIS is named for "SMA(Systems Management Agent" [10].

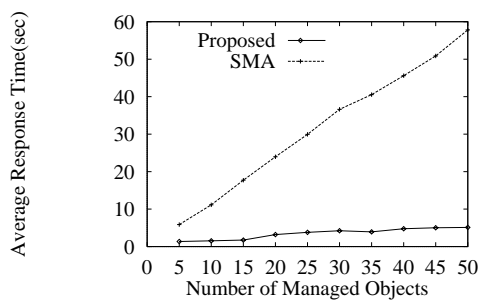

Figure 9. Average response time to an M-GET operation (RPC)

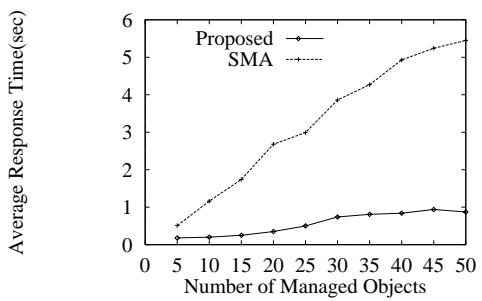

Figure 10. Average response time to an $M$ SET operation (RPC)

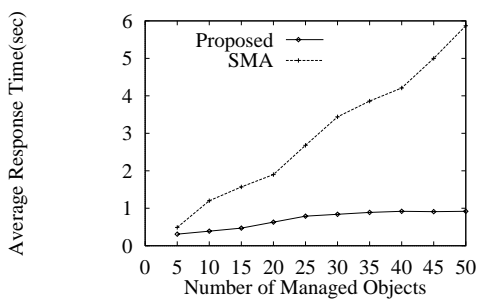

Figure 11. Average response time to an $M-$ ACTION operation (RPC)

Figure 9 shows an average response time to an M-GET operation both for Proposed and for SMA. The horizontal axis means "the number of selected managed objects" and the vertical axis "an average response time to an operation". While the average response time of SMA is proportional to the number of selected managed objects, Proposed is less affected by that. Performance of Proposed is about $4.3 \sim 11.3$ times faster than that of SMA. Similar results can be seen in Figure 10 and 11 which show the average response time to an M-SET/M-ACTION operation, respectively. Performance of Proposed for an M-SET operation is about $2.3 \sim 6.3$ times faster than that of $S M A$, and for M-ACTION operation, $1.6 \sim 6.4$ faster than that of $S M A$. As the number of managed objects increases, performance of our system which concurrently performs a CMIS operation is much better than that of OSIMIS which sequentially performs a CMIS operation. In Figure 9, 10, and 11, when the number of managed objects is the same, it takes longer time to perform an M-GET operation than an M-SET/MACTION operation. This is not a general case and limited to our unixHost class implementation.

\subsection{Communication protocol: IPC}

Figure 12 shows a management system configuration which monitors the state of processes within a UNIX system. We defined a managed object class process which represents a UNIX process. Figure 13 shows an average response time to an M-GET operation. Performance of Proposed is not so good with respect to $S M A$, even worse when the number of selected managed objects is less than 10 . Because communication delay of IPC is shorter than that of RPC, we cannot benefit greatly from multiple threads.

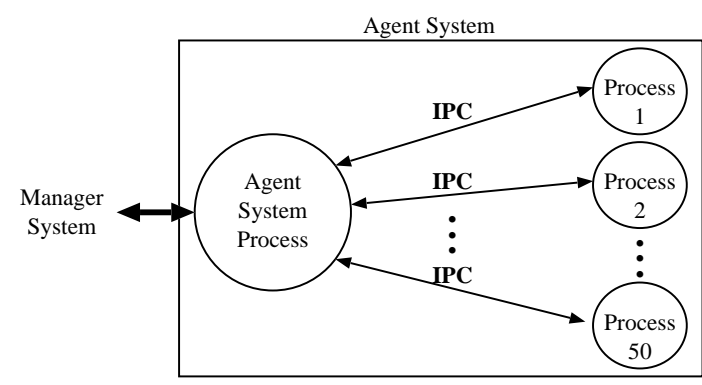

Figure 12. System configuration when IPC is used as a communication protocol

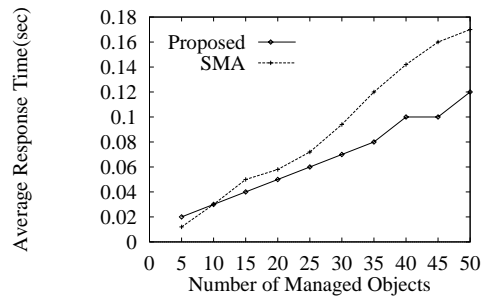

Figure 13. Average response time to an MGET operation (IPC) 


\subsection{Communication protocol: system call}

Figure 14 shows a management system configuration which manages file table inside operating system kernel. We defined a managed object class file which represents a file table entry using GDMO. Figure 15 shows an average response time to an M-GET operation. Note that performance of Proposed is worse than that of SMA. Since delay of a system call is so short that there are more overheads than performance improvement we could expect from multiple threads.

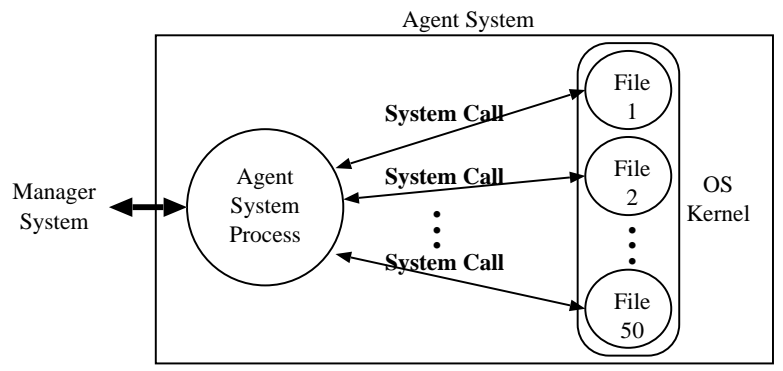

Figure 14. System configuration when system call is used as a communication protocol

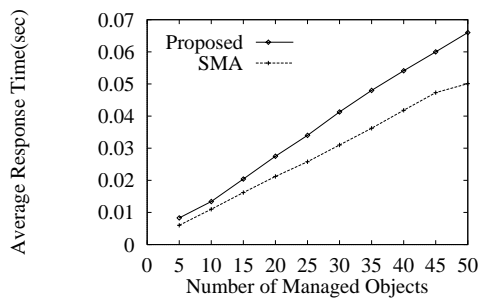

\section{Figure 15. Average response time to an M- GET operation (system call)}

\section{Conclusions}

In this paper, we designed and implemented an agent system which efficiently performs CMIS operations. While existing agent systems sequentially perform a CMIS operation on selected managed objects, our system concurrently performs by creating a thread for each managed object. To resolve mutual exclusion problem caused by multithreading, we used a thread manager which guarantees only one thread can access the same managed object at a time. Since the coordinator thread does not get blocked, our agent system will never get blocked.
From experiments, we can conclude that performance of our system is dependent on the communication protocol used in managed objects: performance of our system is worse than that of OSIMIS when system call is used, slightly better when IPC is used, and much better when RPC is used. We sugguest that threads be used for the following cases: when a network resource resides outside the agent system or although a network resource resides within the agent system, communication delay is long enough to gain benefit from multiple threads.

\section{References}

[1] U. Black. Network Management Standards. McGraw-Hill, Inc., 1995.

[2] CCITT Recommendation X.710. Common Management Information Service Definition. ISO/IEC 9595, 1991.

[3] CCITT Recommendation X.711. Common Management Information Protocol Specification. ISO/IEC 9596, 1991.

[4] CCITT Recommendatoin X.701. Systems Management Overview. ISO/IEC 10040, 1992.

[5] J. Celestino and J.-P. Claude. Building a Platform Support Environment for TMN Applications. In IEEE ICC'94, 1994.

[6] B. Hebrawi. OSI Upper Layer Standards and Practices. McGraw-Hill, Inc., 1993.

[7] ITU-T. Recommendation M.3010 - Principles for a Telecommunications Management Network. ITU-T, 1992.

[8] G. Knight, G. Pavlou, and S. Walton. Experience of Implementing OSI Management Facilities. In International Symposium on Integrated Network Management, 1991.

[9] S. Nakai, Y. Kiriha, Y. Ihara, and S. Hasegawa. A Development Environment for OSI Systems Management. In International Symposium on Integrated Network Management, 1991.

[10] G. Pavlou, K. McCarthy, S. Bhatti, and G. Knight. The OSIMIS Platform: Making OSI Management Simple. In International Symposium on Integrated Network Management, 1995.

[11] M. T. Rose, J. P. Onions, and C. J. Robbins. The ISO Development Environment User's Manual version 7. PSI Inc./XTel Services Ltd., 1991.

[12] Y.-C. Shim. Developing a Managed System in a Telecommunication Management Network. In IEEE ICC'96, June 1996.

[13] UCL. A Guide to Implementing Managed Objects Using the GMS, 1992.

[14] UCL. The OSI Management Information Service User's Manual, 1992. 